INTERIM REPORT

U.S. Department of Energy

\title{
HIGH FREQUENCY ELECTROMAGNETIC IMPEDANCE MEASUREMENTS FOR CHARACTERIZATION, MONITORING AND VERIFICATION EFFORTS
}

\author{
Principal Investigators: \\ Ki Ha Lee \\ Lawrence Berkeley National Laboratory \\ 1 Cyclotron Road, MS 90-1116 \\ Berkeley, CA 94720 \\ (510) 486-7468, (510) 486-5686 (fax) \\ khlee@lbl.gov \\ Alex Becker \\ University of California, Berkeley \\ Berkeley, CA 94720 \\ alexb@socrates.berkeley.edu
}

Project Number: 60328 (renewed to 73776)

Grant Number: DE-AC03-76SF00098

Grant Project Officers: Dr. Mark Gilbertson and Dr. Roland Hirsh 
Project Duration: 1997 - 2003 (including the renewal) 


\section{Table of Contents}

\section{Cover Sheet}

Table of Contents

Executive Summary

2

Research Objectives

4

Methods and Results

6

Relevance, Impact, and Technology Transfer

Project Productivity

1) Field Measurement Techniques

13

2) The Field-Hardened Prototype HFI System

14

3) Data Processing, Analysis, and Interpretation

Personnel Supported

Publications

Interactions

Transitions

Patents

Future Work

18

Literature Cited

18 


\section{Executive Summary}

Non-invasive sensing of the shallow subsurface is necessary for detection and delineation of buried hazardous wastes, monitoring of the condition of clay containment caps, and a variety of other purposes. Electromagnetic methods have been shown to be effective in environmental site characterization, but there is a need for increased resolution for waste form characterization, verification, and monitoring activities. A window exists in the electromagnetic spectrum between ground penetrating radar frequencies (30 MHz to $1 \mathrm{GHz})$ and induction technique frequencies $(<100 \mathrm{kHz})$ that has not been utilized for these applications. Two considerations strongly suggest the use of frequencies in this band for such applications: 1) the induction response of many targets of environmental concern is small due to small size, and 2) a need to determine both the electrical conductivity and dielectric permittivity which are related to shallow subsurface geochemistry and geohydrology. Modeling and physical parameter studies confirm that measurements at frequencies between 1 and $100 \mathrm{MHz}$ can yield useful resolution of subsurface conductivity and permittivity. The term "high frequency" as used herein refers to this frequency band.

Application of high-frequency electromagnetic methods to shallow subsurface investigations can best be accomplished using the impedance approach. The impedance is the ratio of the electric to magnetic fields, so the source information is not necessary in the analysis stage. We proposed to develop and demonstrate proof-of-concept of the high-frequency impedance (HFI) methodology by field testing and verifying the performance of off-the-shelf equipment, and by evaluating the basic capability for interpreting HFI data for both the electrical conductivity and permittivity. The proof-ofconcept for the HFI system has been demonstrated in the first phase of the research program (1997 
through 2000). Field tests were done in both the relatively conductive environment and far more resistive ones. For the first time both the electrical resistivity and permittivity of shallow subsurface have been analyzed together with encouraging results. The successful integration and field tests are as a result of the three-year research work consisting of careful evaluation of off-the-shelf electric and magnetic field sensors, conducting theoretical model studies, acquisition of test gears for proof-of-concept system, and describing specification of HFI system.

Calibration of sensors, HFI system integration, and field tests have been conducted by Dr. William Frangos under the supervision of Professor Alex Becker of University of California, Berkeley. Dr. Frangos' work on this project has been summarized in his doctoral dissertation titled 'High Frequency Impedance Measurements for Non-invasive Permittivity Determination.' An important accomplishment of his work is that measured impedances agree with the calculated ones for a layered earth structure constructed using independently measured resistivity and estimated permittivity values. Impedance data from a resistive environment, more representative of clay caps at Savannah River Site (SRS), also agree with forward modeling results of the known resistive structure and yield reasonable estimates of the permittivity. Copy of Dr. Frangos' thesis may be available from Professor Alex Becker (alexb@socrates.berkeley.edu). 


\section{Research Objectives}

Electromagnetic methods in exploration geophysics include many technologies capable of imaging the subsurface. The electromagnetic geophysical spectrum for shallow subsurface imaging is roughly $1 \mathrm{~Hz}$ to $500 \mathrm{MHz}$, with electrical resistivity and other geometric sounding methods located at the low frequency end and the familiar GPR method at the high end of the spectrum. Baseline studies (Pellerin et al., 1997) show that electromagnetic instrumentation in the mid- and low-frequencies (<300 $\mathrm{kHz}$ ) and GPR systems (> $30 \mathrm{MHz}$ ) are well developed in the commercial sector. In the high-frequency range of $300 \mathrm{kHz}$ to $100 \mathrm{MHz}$ developments have been quite recent and reside within the research community. Accurate theoretical numerical modeling algorithms are available for simulations and interpretation across the entire spectrum (Mackie and Madden, 1993; Pellerin et al., 1995; Pellerin et al., 1997; Alumbaugh and Newman, 1995; Lee et al., 1995, Newmann and Alumbaugh, 1997; Newmann, 1999; Sasaki, 1999, etc.), but instrumentation suitable for collecting calibrated field data in the important high-frequency range is critically lacking. Several attempts to develop reliable, accurate and calibrated instruments (Sternberg and Poulton, 1996; Stewart et al., 1994; Wright et el., 1996) have produced mixed results.

We proposed to exploit the concept of electromagnetic impedance, the ratio of orthogonal horizontal electric to horizontal magnetic fields, to provide the necessary technology in the highfrequency band described above. The effective depth of investigation for surface impedance measurements depends on the frequency, and is commonly expressed in terms of the skin depth, the distance into the conductive half space at which the amplitude of the incoming wave has decreased to $e^{-1}$ of its surface value. In order to achieve skin depths between 0.5 and 10 meters in material of resistivity between 1 and $100 \mathrm{ohm}-\mathrm{m}$ and relative permittivity between 1 and 30, frequencies between about 300 $\mathrm{kHz}$ and $100 \mathrm{MHz}$ are required. To achieve better resolution in permittivity, we need to utilize data at frequencies at the high-end of this spectrum. It is also generally true that resolution in permittivity can best be realized in an area of high electrical resistivity.

To emphasize the utility and the importance of high-frequency EM measurements for mapping subsurface distribution of moisture content, we take as example a clay cap model. Figure 1 presents a simple clay cap application, based on conditions at the Savannah River Site H-Area Basin, where the specific problem is the shrinkage-induced cracks in the clay cap as it dries. Measured resistivities are about $500 \Omega-\mathrm{m}$ (Persoff, et al., 1996). The relative permittivity calculated from an experimentally validated standard mixing law (Knoll, 1996) for a clay-air-water mixture ranges from 17 or greater for a healthy clay to 12 and less for one which is too dry for regulatory compliance. Thicknesses and expected permittivities are shown for all layers of the cap. The surface 'plane-wave' impedance, in ohms, is plotted against frequency. As is apparent, the good and bad clay conditions manifest themselves in a $27 \%$ difference in amplitude at about $30 \mathrm{MHz}$. Adjusting only the layer resistivities within the ranges observed at the site however, perturbs these curves by less than $5 \%$. The same geometry with the observed resistivities and equal permittivities yields a nearly featureless spectrum. The change in impedance is caused solely by permittivity contrast, which cannot be detected by any conductivity-sensitive method. Variation of the clay water content yields little change in resistivity, since surface conduction dominates over saturation effects. A ground penetrating radar (GPR) survey did not 
result in clear images in tests done at a nearby site composed of the same clays and soils. Clearly, quantitative knowledge of the layer permittivity is required to solve the problem.
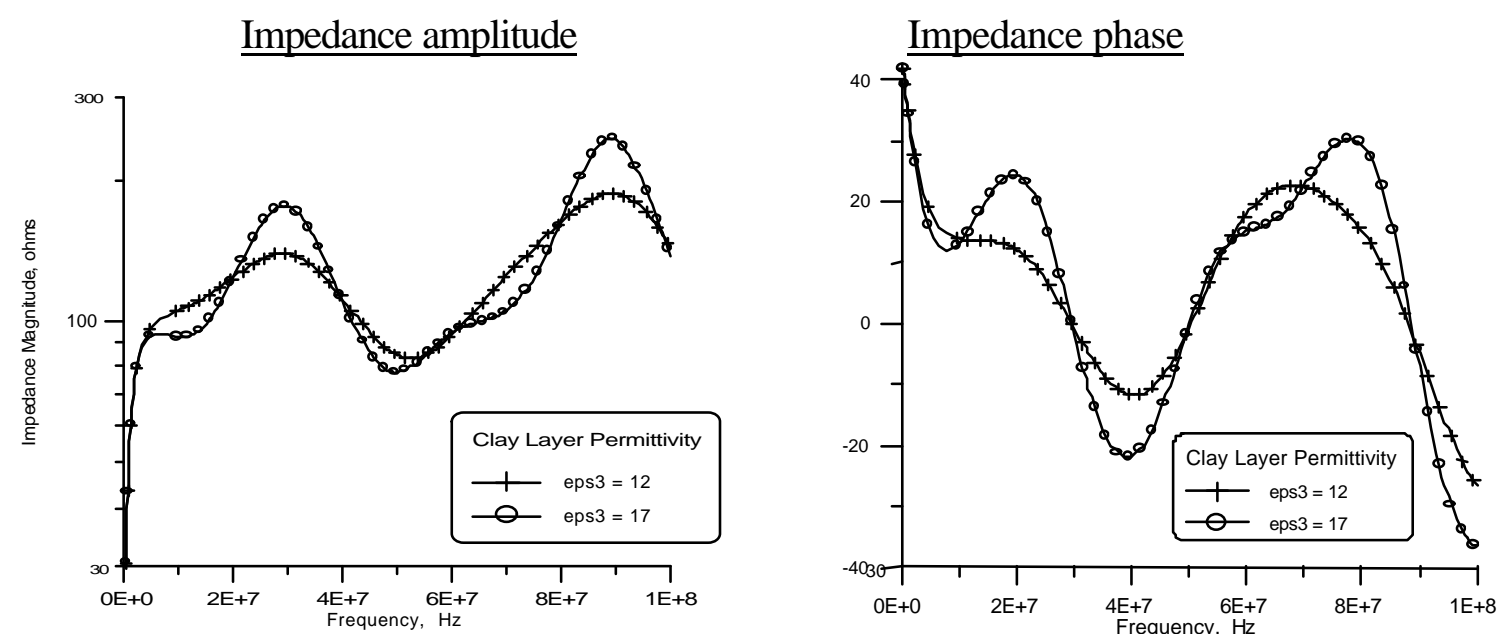

Air, grass, and vegetation

\begin{tabular}{lll}
\hline Top soil & $_{1} \bullet 10$ & $0.61 \mathrm{~m}$ \\
\hline Gravel & 2 $_{2} \cdot 5$ & $0.23 \mathrm{~m}$ \\
\hline Clay & * $_{3} \bullet$ var. & $0.61 \mathrm{~m}$ \\
\hline Soil & * $_{4} \cdot 10$ &
\end{tabular}

Previous efforts in developing a high-frequency controlled-source, electromagnetic system have encountered problems in system calibration. Instrument gain drift was addressed by Sternberg and Poulton (1996) and Stewart, et al. (1994) by transforming the received signal into ellipticity and tilt. These parameters are difficult to use in inversion type interpretational schemes. A more significant problem discovered during extensive analysis of the system developed by Stewart et al. (1994) may involve spurious coupling of the system to the earth so that the effective system transfer characteristics vary from site to site resulting in uncalibrated data. These systems can only function as an anomaly hunters and cannot provide data suitable for producing quantitative images of the subsurface.

Extensive testing of the instrument developed by Stewart et al. (1994) made it possible to document the problems described above and develop an alternative approach to system design (Pellerin et al., 1996). The system of Sternberg and Poulton (1996) uses a neural network for the interpretation of ellipticity data. This alleviates many problems, but there's is a unique system that is only capable of finding targets for which it has been trained. The very early time-domain instrument of Wright et al. (1996) is quite rapid in data acquisition, but probably suffers from the transmitter loading problems 
because it has the same geometry as the other systems. It is also quite sensitive to slight variations in transmitter-receiver orientation, and the availability of simulators and interpretational algorithms is limited. Another controlled-source EM radio imaging (RIM) system has been developed between different entities in the commercial and academic sectors with disappointing results (Ken Mahrer, RIMtech, personal communication; 1994; Greg Moran, New Mexico State Univ., personal communication, 1995, 1996). Our proposed HFI system generates EM impedance data; i.e., the electric to magnetic field ratio. This ratio is only a function of source position and the nature of polarization, so it is not necessary to know the source function itself, a big advantage in data acquisition. Furthermore, if the source-receiver distance is great enough, or the frequency is high enough, the EM impedance is identical to the plane-wave impedance. In this case we can utilize the well-developed MT interpretation methods with minor modifications.

There are several advantages in the field ratio approach over the measurement of individual field approach employed by the USGS HFS and the University of Arizona high-frequency ellipticity system. As mentioned earlier, an extensive system analysis of the USGS HFS showed that at the frequency of interest the source cannot be well defined and hence cannot be accurately modeled, as is essential for quantitative interpretation. A field ratio approach does not require information about the source function, nor any absolute phase reference.

Interpretation of EM impedance data, in general, requires the inversion of EM fields for electrical parameters. Many studies have been presented for the inversion of EM fields in terms of electrical conductivity distributions, but there is lack of work dealing with the simultaneous inversion of electrical conductivity and permittivity using a single data set of measurements. One very important aspect of the proposed research is to address this issue using simple 1-D problems to begin with.

\section{Methods and Results}

A prototype 0.1 to $30 \mathrm{MHz}$ system was assembled using off-the-shelf components including a magnetic dipole transmitter, electric and magnetic antennae. Three fiber-optic coupling systems were used to achieve the necessary isolation between source and receiver. Magnetic and electric field sensors are typically positioned at a height of about one meter. The prototype HFI system components

are shown in Figure 2. Field tests were done in the relatively conductive (20 to $50 \mathrm{U}-\mathrm{m}$ ) environment of the University of California's Richmond Field Station and a far more resistive ones (2,000 to 10,000 Ù$\mathrm{m})$ at the Point Reyes National Seashore and Donner Summit. Encouraging results were obtained at all the sites. Some of the results are summarized in this report. 
High Frequency Electromagnetic

Sensors

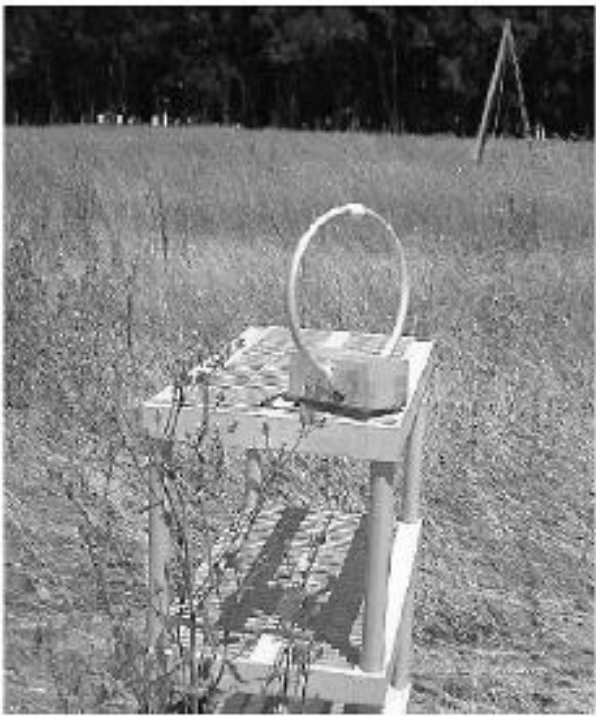

(A)

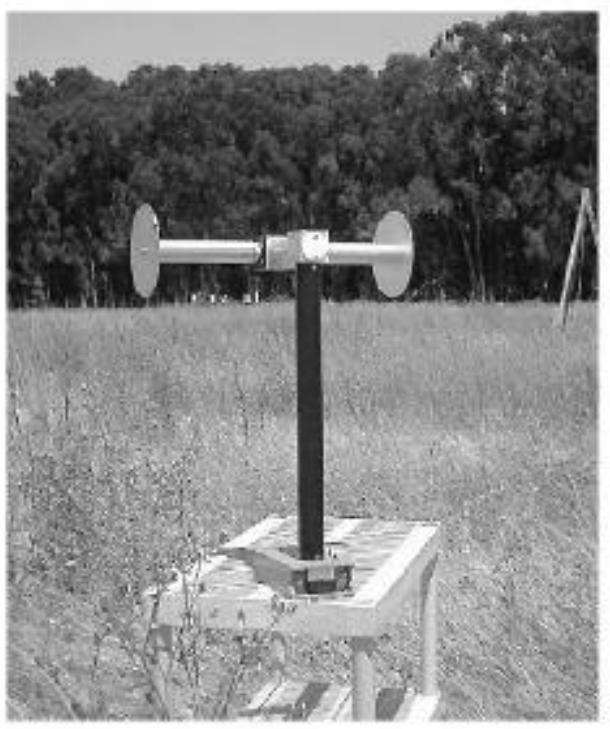

LBNL High Frequency Impedance Project DoE EMSP \#60328

(B)

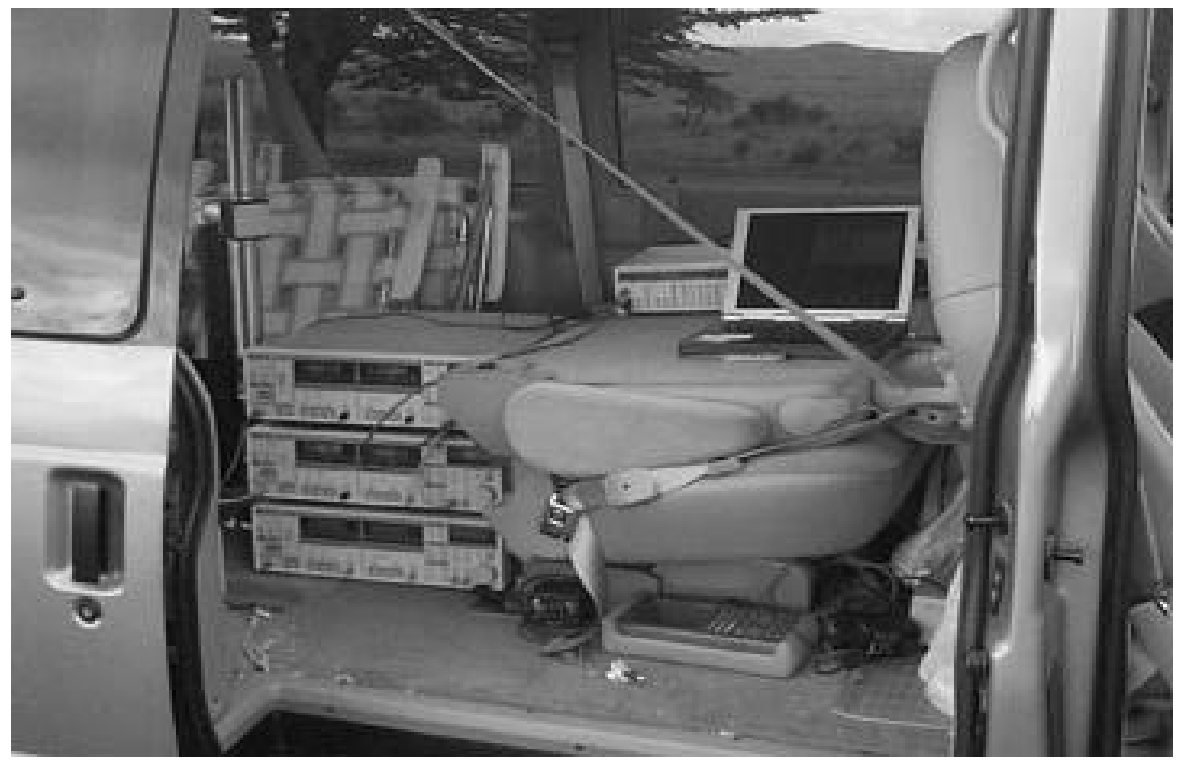

(C)

Figure 2. HFI system components. (A) loop magnetic sensor, (B) stub electric field antenna, and $(\mathrm{C})$ measuring equipment within a rented minivan. 
Figure 3 below shows observed and fitted data from the Richmond site, taken with a transverse magnetic dipole (My) source. A DC resistivity sounding at this location shows a resistive, 0.4-m upper layer of about $200 \mathrm{ohm}-\mathrm{m}$ overlying a half space of about $40 \mathrm{ohm}-\mathrm{m}$, which is in good agreement with the interpreted model. The permittivity is not well resolved due to the low resistivity. These data demonstrate that the high frequency measurements are accurate since they do include the independently determined layered resistivity structure. The permittivity values were unverified but appear reasonable for the test environment.

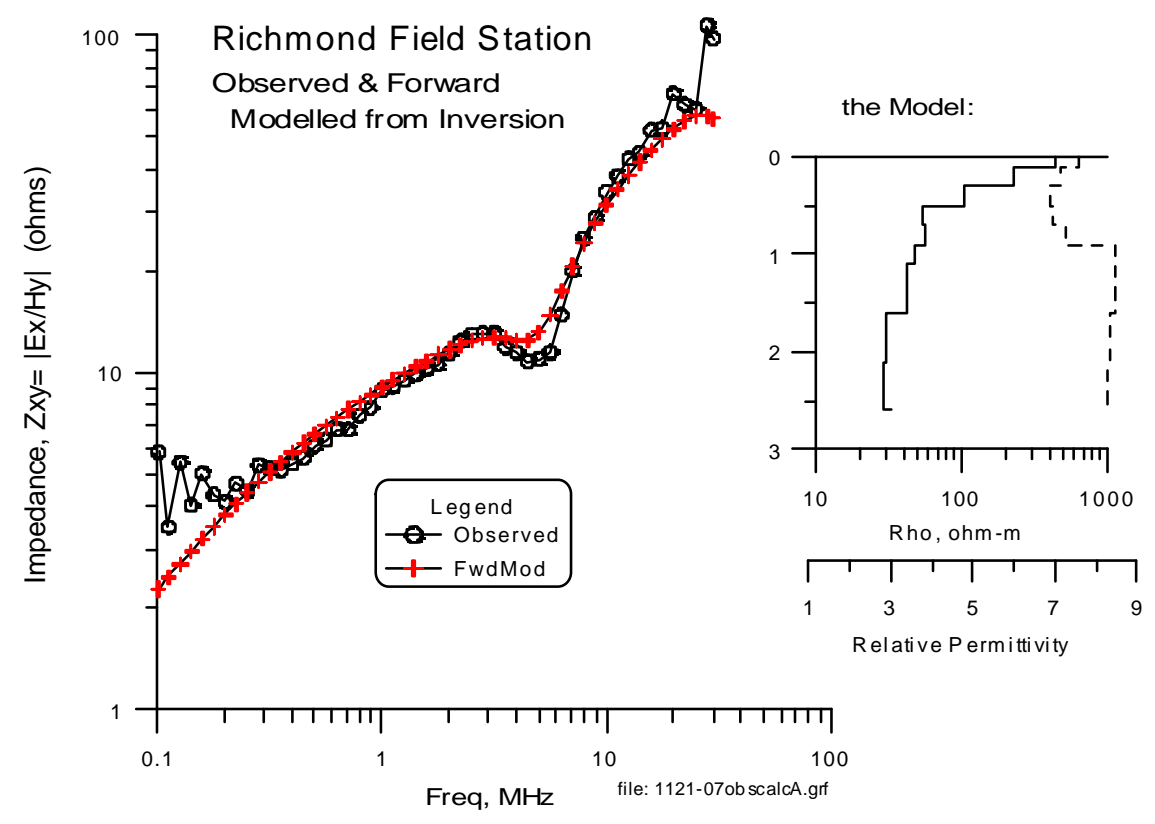

Figure 3. Data obtained at the Richmond Field Station and fitting with 1-D inversion (on the right). Horizontal magnetic dipole (My) was used as source. Inversion was done using Occam's algorithm

Inversion of EM impedance for conductivity and permittivity was carried out using 1-D models. We assume that the earth consists of $N$ discrete layers, each of which is characterized by its electrical conductivity $S_{i}$ and electrical permittivity ${ }_{i}$. We further assume that each layer has its thickness $h_{i}$, and that the magnetic permeability is homogeneous with its value the same as for free space. With these assumptions, an initial test for simultaneous inversion for electrical conductivity and permittivity was made in 1999 (the second year of the first phase project). In case the source used was a magnetic dipole polarized in the $y$-direction, $H_{y}$ and $E_{x}$ field measurements were made for the impedance calculation. If the source was polarized in the $x$ - or $z$-direction, then measurements were made for $H_{x}$ and $E_{y}$. Typical separation between transmitter and receiver was $8 \mathrm{~m}$. The inversion of impedance 
data was carried out based on the Occam's algorithm using the numerically evaluated sensitivity functions. If the impedance is $Z_{x y}\left(E_{x} / H_{y}\right)$, then the sensitivity is evaluated by a differencing scheme

$$
Z_{x y}^{p_{i}}=\frac{\partial}{\partial p_{i}} Z_{x y}=\frac{Z_{x y}\left(p_{i}+D p_{i}\right)-Z_{x y}\left(p_{i}\right)}{D p_{i}}
$$

with $p_{i}$ being either the conductivity or the permittivity of the $i$-th layer at each iteration. The differencing scheme adopted here is a good approximation as the parameter increment approaches zero, but the accuracy in representing the true sensitivity function may be questionable when the impedance changes rapidly as a function of the given parameter. This is a valid concern especially when we are dealing with EM waves that propagate rather than diffuse.

Next, in Figure 4, we show the observed and calculated data from the Abbot's Lagoon site located on the Point Reyes National Seashore. In this case, the permittivity is well resolved, since permittivity dominates the response, as shown by modeling analysis using the 1-D code (EM1D). 1-D inversion was carried out and the resulting model was used to obtain the calculated impedance data. This set of circumstances is more representative of the Savannah River Site (SRS) clay cap situation, a resistive environment, the original problem that prompted this research. By mapping permittivity, we may determine moisture content which, in turn, relates to the engineering condition of the clay caps. The interpreted model provides a good fit to the data, however, the model is somewhat different from the information obtained using other independent means such as dc resistivity and GPR (Figure 5).

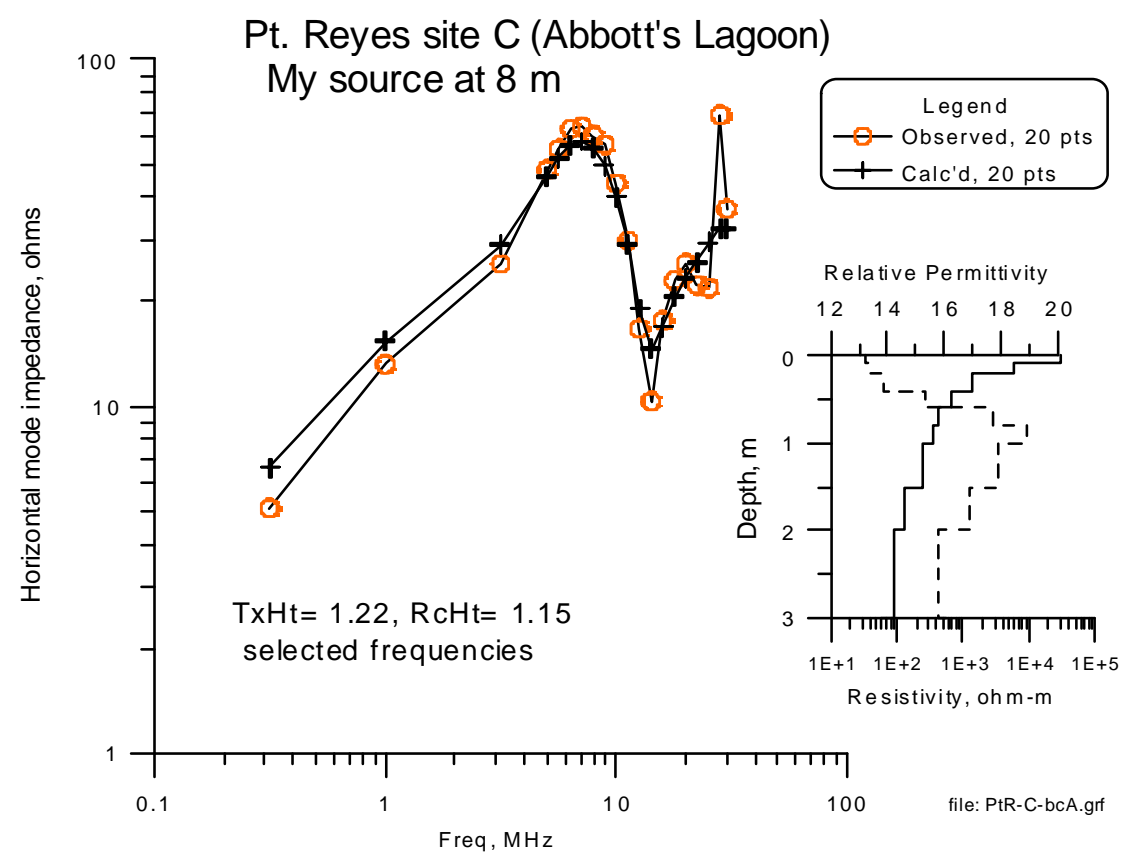

Figure 4. Impedance data $\left(Z_{x y}\right)$ obtained at Pt. Reyes. 1-D inversion is shown on the right. Solid line is the resistivity, dotted line the relative permittivity. 


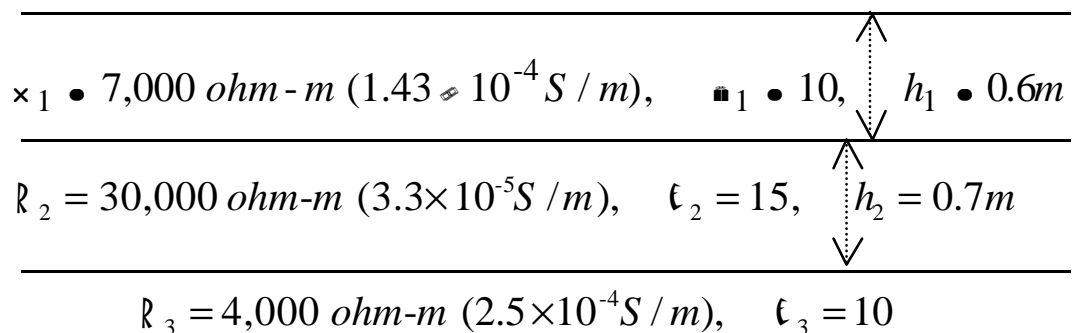

Figure 5. A 3-layered earth suggested by dc resistivity and GPR data.

This exercise with real data in simultaneously reconstructing subsurface conductivity and permittivity distributions offers valuable lessons. First of all, it is necessary to evaluate the sensitivity functions correctly. Secondly, we may need to consider an inversion scheme in which parameters with very different sensitivity characteristics can be effectively dealt with. Finally, it may be necessary to sample frequencies linearly where the EM fields behave as propagating wavefields.

Another set of data, taken with a vertical magnetic dipole source, is displayed below in Figure 6. The fitted model in this case is a simple homogeneous earth with resistivity of 2,000 ohm-m and relative permittivity of 5. The fit is not as good as other examples shown above, because the model used to fit the data is a simple homogeneous half-space obtained by trial and error, and not through the inversion. Nevertheless, the fit at frequencies above $10 \mathrm{MHz}$ is striking, and this confirms the performance of the prototype HFI system in detecting the effect of electrical permittivity of the subsurface.

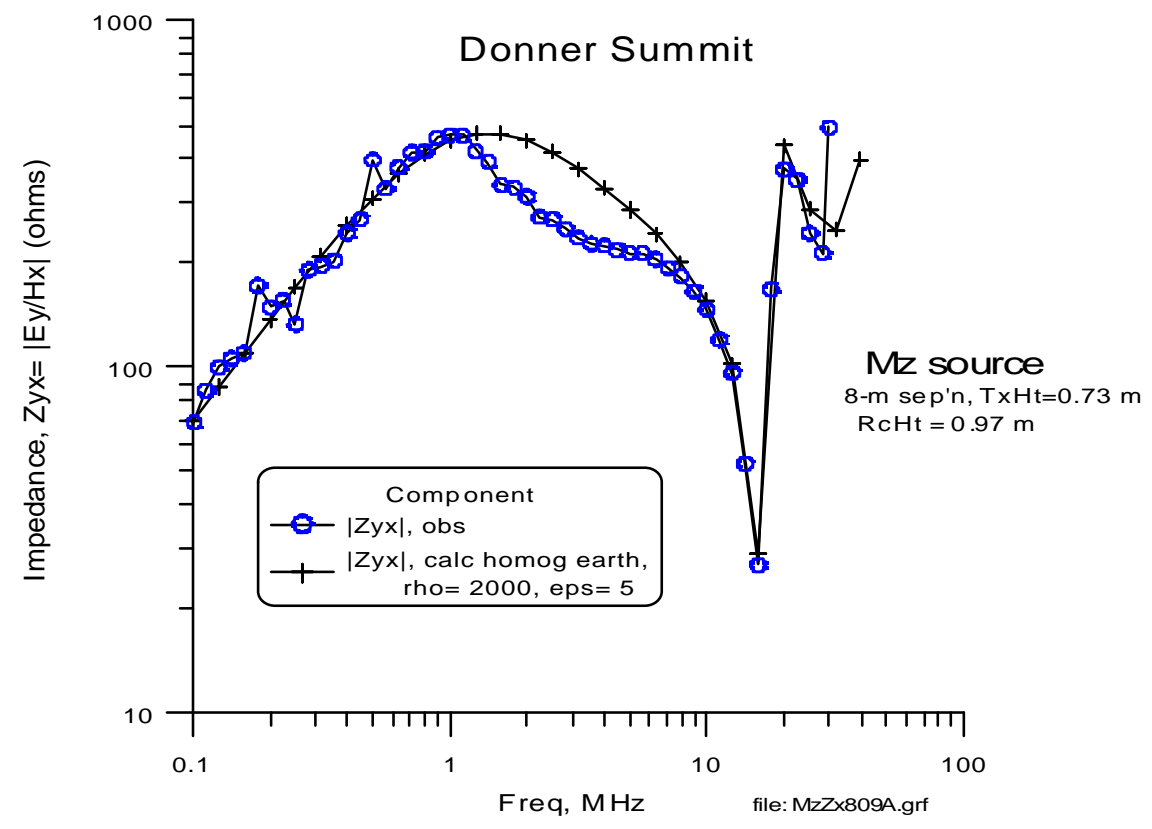

Figure 6. Data obtained at the Donner Summit and fitting with homogeneous 
half-space model. A vertical magnetic dipole was used as source.

As an on-going sensor technology development, measurement of the electric field using a toroidal sensor was continued. The toroid used for the test and the antenna factor spectrum (terminal voltage divided by the ambient electric field) derived from the measurement are shown in Figures 7 and 8 , respectively. The sensor shows spurious effects in its frequency response as it exhibits a number of undesirable local resonances.

Figure 7. Toroidal coil (actual size). . The total size of the toroid is 3" and the diameter of the cross section is 1". The number of turns wound around the toroid is total 100 turns. For the testing, the windings were shielded using copper strips to reduce the extraneous pickup and capacitive leakage.

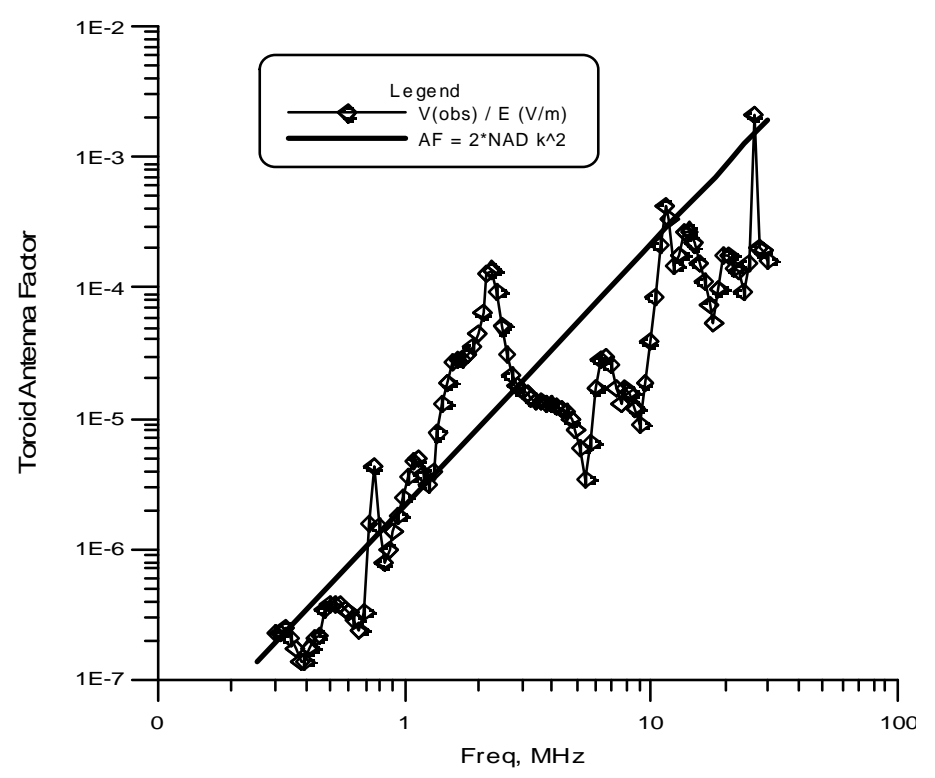


Figure 8. Measured (diamonds) and calculated (solid line) response spectra

for the toroidal antenna.

\section{Relevance, Impact, and Technology Transfer}

Characterization technologies are necessary at all levels of the EM restoration effort. Geophysics has been an important player in site characterization and will have an increasing role in verification and monitoring activities (e.g., STCG\# ID-S.1.04, AL-09-01-03-SC-S; Subsurface Contaminants Focus Area). Electromagnetic geophysical methods can yield estimates of conductivity and permittivity (e.g., STCG \#. RL-WT045-S, Tanks Focus Area) that are very useful in environmental investigations. High resolution techniques are particularly needed for the following DOE applications:

- long-term monitoring the condition of clay caps,

- monitoring of vadose zone groundwater and moisture contents,

- characterization of landfills, buried waste pits and trenches,

- delineation of contaminant plumes,

- verification and monitoring of an engineered subsurface barrier,

- monitoring of grout injection for stabilization and containment, and

- location of contaminant source terms such as non-aqueous phase liquids.

To achieve high resolution, spatially and spectrally dense measurements of high-frequency electromagnetic fields are essential. Commonly optimal system response to the conductivity and dielectric parameters as a function of depth is located in this range. Depths of exploration are shallow $(<$ $10 \mathrm{~m}$ ) in environmental investigations and the terrain is often conductive due to the existence of clay minerals, such as a clay cap over a buried waste site, so that ground penetrating radar (GPR) is ineffective. At low frequencies, on the other hand, array sizes or plane wave skin depths (Vozoff, 1986) are large resulting in a minimum depth of investigation that is too deep and/or the lateral resolution that is too poor to be of general use. Theoretical models show that the electromagnetic response related to the drying or dewatering of a clay cap is detectable in the high-frequency range. It should be noted that in addition to high frequency measurements, dense spatial sampling is necessary for applications such as subsurface barrier verification and landmine detection and that these can be handled efficiently with the proposed measurement system.

\section{Project Productivity}

In the first phase (FY1997-FY2000, Project Number 60328) of the research program, we proposed to develop: 1) high-frequency field measurement techniques, 2) plane wave impedance estimation, and 3) data processing, analysis, and interpretation techniques. We have completed most of these objectives by field demonstrating proof-of-concept of the proposed technology, but it was determined that actual field deployment of thus developed technology to DOE sites will have to wait until further improvements are made to the HFI system. Careful evaluation of the state of the project led us to apply for a renewal of the project for the second phase. As a result, we have recently been 
awarded a renewal with the Project Number 73776, effectively extending the overall project completion to the year 2003 .

The proposed research plan consists of three tasks: 1) continued development and completion of high-frequency field measurement techniques, 2) construction and delivery of a field-hardened prototype HFI system, and 3) data processing, analysis, and interpretation. Construction of a mobile HFI field system can be accomplished using off-the-shelf instrumentation and commercially available components, but research will be continued to develop better high-frequency sensors using innovative designs. Development of an analysis package for processing and interpretation of high-frequency data is also an essential part of the proposed work. Towards the end of the project, the prototype system will be capable of real-time mapping of the electrical conductivity and permittivity using simultaneous inversion in one dimension. Feasibility for implementing higher-dimensional analysis package in field operation will be critically evaluated. Following is a brief summary of the proposed work in the renewed program.

\section{1) Field Measurement Techniques}

One problem that requires careful attention is the nature of the sensors to be employed for accurate measurement of the electric and magnetic fields. An ideal electric or magnetic sensor (or "transfer standard" in antenna and radio engineering parlance) yields a precisely known voltage response at all frequencies and field strengths, does not perturb the field being measured, and is insensitive to other aspects of the environment in which it is placed. A surface impedance measurement requires both electric and magnetic field sensors. In all cases, the sensor must be connected to the measuring and signal analysis equipment via a non-perturbing link; we propose to use a commercially available fiber-optic cable system. Further improvements on the measurement techniques will be made by refinements and careful repackaging of elements, and replacing a few of the elements with smaller and more rugged alternatives.

In the development of field deployable HFI system, we no longer consider the capacitively grounded wire suitable as an electric field sensor. The development of new sensors will be concentrated on the further refinement of the toroidal antenna. The device may be used as transmitter as well, and some detail is given below.

Time-varying magnetic fields induce a voltage in a toroidal coil. The electric field at the center of the toroid is shown to be linearly related to this induced voltage (Lee, 1997). So, by measuring the voltage across a toroidal coil one can easily and accurately determine the ambient electric field. The overall size of the toroidal sensor can be as small as a few centimeters. It is this size advantage that not only allows easy fabrication and deployment of multi-component devices, but also may render greatly improved spatial resolution over conventional systems.

In the first phase of our research project a simple air-core toroid of small diameter was manufactured (Figure 7 in Methods and Results section) to test and verify the basic concept involved. A functional comparison between the stub and toroid sensors has also been made. The longest 
dimension of each sensor is $5 \mathrm{~cm}$ and the toroid is presumed to have an air core. The toroid has a weaker sensitivity at all but the highest frequencies and a higher source-impedance in the higher portion of the spectrum.

An interesting proposal was presented to use the toroidal coil as a vertical electric dipole transmitter (Wait, J.R., 1995). In this proposal, the toroidal coil is excited by a current of strength $I$, resulting in a ring of 'magnetic' current. The magnetic current is then related to an equivalent electric dipole, $I_{s} d s$, located at the center of the toroid. Following this idea, the proposed research will include the feasibility study of using the toroidal coil as a transmitter. The advantage of using such a device as transmitter is obvious; it is small in size, so that deployment of such a device will result in better spatial resolution. Furthermore, this type of device is ideal for borehole high-frequency EM surveys. Conventional EM tools, including GPR, cannot be used efficiently when deployed in the borehole because of the size limitation. Nor can they be used to generate horizontal electric field.

\section{2) The Field-Hardened Prototype HFI System}

The presently available proof-of-concept equipment is not well suited for extensive field experimentation. A more mobile system must be assembled by repackaging the basic components and replacing a few of the more provisional ones with smaller, more rugged alternatives. We propose to build a measurement system that maintains the relative source and sensor positions as it is towed or pulled across a survey area, which can be broken down for shipment by air and readily carried in a minivan. A control module will house the computer, lock-in amplifiers, function generator, and the three fiber optical systems. The sensors and the transmitter system will be mounted on wheels using a rigid, non-conducting frame. A sketch of the contemplated cart is shown below.

\section{Sketch of proposed field-hardened prototype HFI system}

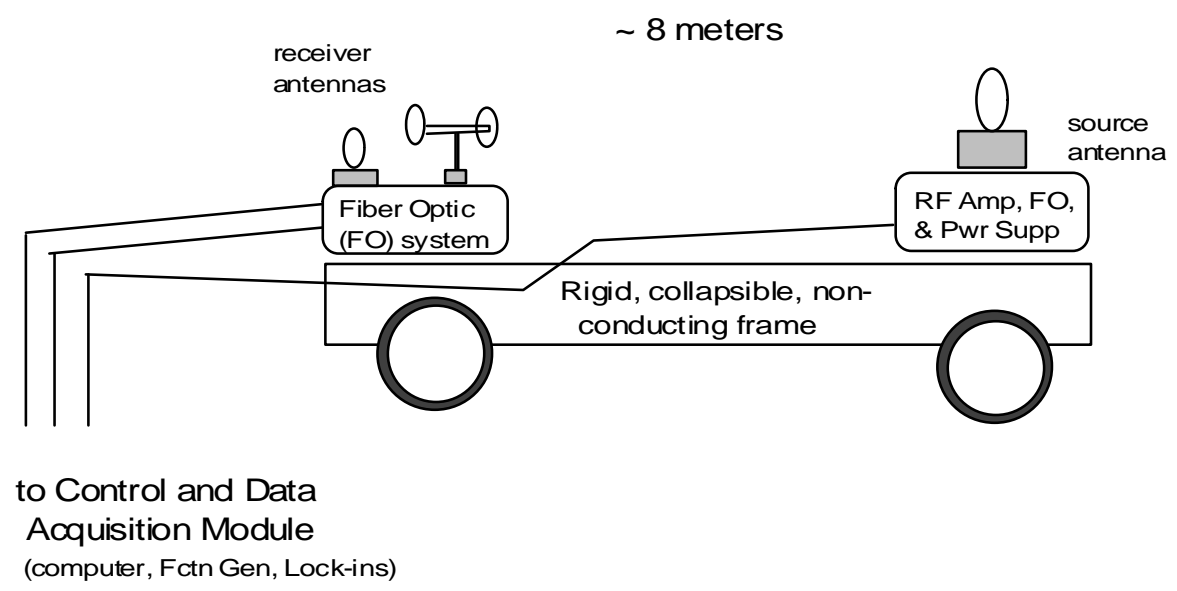

file: cartplot.grf 
Figure 9. A computer-controlled, optical fiber connected prototype HFI system

The repackaged system is envisioned in three modules, one each for the transmitter, the receiver, and the control functions, plus the cart. The transmitter module will contain the source antenna, an RF amplifier, a fiber-optic receiver, and a power supply. The receiver module will mount the two field sensors (electric and magnetic) and two fiber-optic transmitters; power requirements are minimal and should be handled by small batteries for each individual unit to maintain isolation. The control module will host the control computer, function generator, lock-in amplifiers, two fiber optic receivers, one fiber optic transmitter, and a power supply. In operation, the computer sets the function generator frequency (and perhaps amplitude); the output is supplied to the fiber optic transmitter for transmission to the transmitter module and to the lock-ins as a phase reference. After an appropriate settling time, the computer reads and records the amplitudes and phases of the electric and magnetic fields from the lock-in amplifiers (perhaps after modifying the gain or settling time settings).

The fiber optic system currently in use is a general-purpose type and is no longer manufactured; soon it will not be supported. It should be replaced with a smaller and more robust system, tailored to the application. A number of commercial, multi-channel fiber optic systems are currently available, many marketed for closed-circuit video applications. These devices include the essential components of an instrumentation isolation system (modulated laser transmitter, demodulating optic receiver), but do not meet our requirements in off-the-shelf form. We propose to adapt a commercial system to the battery-powered, calibrated form necessary for use in the HFI system. This step will undoubtedly require some testing and re-engineering of several candidate systems.

Simultaneous acquisition of two or more data channels, electric and magnetic, will result in a mobile survey system, capable of continuous data acquisition while being towed, for example. An important issue to be decided concerns the spectral resolution necessary for various targets and survey styles. If only a relatively few frequencies are needed to solve a particular problem, then surveying might proceed in a continuous or near-continuous mode, while a more dense spectral sampling would require a station-by-station style of data acquisition. System positioning will be done with differential GPS.

3) Data Processing, Analysis, and Interpretation

The key objective of this proposal is the simultaneous mapping of the electrical conductivity and permittivity of the near surface earth. Ideally we planned to adapt classical MT plane wave analysis to the high frequency region. Once we were able to make accurate field measurements however, it became evident that the ambient EM noise levels precluded the use of any artificial source that can be placed sufficiently far from the receiver so as to observe plane wave propagation. Any source with sufficient strength to allow accurate observations at distances on the order of a wavelength would contravene FCC regulations. Not only would such a source be illegal but also the risk of interfering with essential communication services must certainly be avoided. Thus, by force of circumstance we are led to use a relatively short source receiver spacing and to develop and perfect interpretation techniques 
suitable for dealing with EM impedance data. Although this change in measurement technique adds a certain degree of complexity to the data interpretation, it brings with it the possibility of evolving a highly mobile and compact instrumentation system.

Because of these reasons, and the relative simplicity involved in dealing with 1-D EM inversion we plan to use 1-D inversion of EM field data as a primary tool for mapping subsurface resistivity and permittivity distributions. The fact that 'impedance' data will be used instead of the individual field data remains unchanged in the proposed work, and the advantage in dealing with the field ratio over any individual field quantity is also retained. Since we are now considering EM impedance, where the individual field is not a plane wave, the impedance is a function of the source-receiver separation, the type of source used, and locations that source and/or receiver occupy.

The 1-D inversion scheme we used to analyze impedance data shown in the Methods and Results section is based on the sensitivity estimated by a differencing scheme. To improve the inversion we plan to use more accurate sensitivity function, including the analytic one.

In the proposed inversion scheme we assign a constant layer thickness of $h$ for all layers, so only the electrical conductivity and permittivity in each layer are inversion parameters. This approach may be reasonable if we choose many small layers to simulate the half space. The simultaneous inversion of electrical conductivity and electrical permittivity may require balanced treatment of each of these parameters in the inversion process. Our approach will be similar to the one presented by Zhang and Oldenburg (1999).

Ultimately, the HFI technology will be extended to include mapping of 3-D subsurface. The task will be critically evaluated once the $1-\mathrm{D}$ problem is under control to the extent that field data can be routinely interpreted in pseudo real-time basis. This requires understanding of the physics and inversion processes of dealing with two distinct electrical parameters. Our preliminary investigation indicates that the inversion of electrical conductivity and permittivity may best be handled sequentially, especially for higher-dimensional problems. This finding is based on the numerical modeling analysis using simple 1-D models, and field examples we have encountered using the HFI system. The field test at Richmond Field Station is completely dominated by diffusion (Figure 3), and at Pt. Reyes by wave propagation (Figure 4) at high frequencies. Typically, it can be observed there is a clean separation in frequency between EM fields dominated by diffusion and wave propagation. The frequency at which this separation takes place is called the transition frequency ${ }_{t} t$ given by 


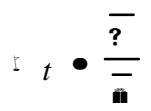

where the bar indicates average of the parameter.

This interesting property can be used in the development of inversion algorithms for imaging both parameters, separately. First, the conductivity distribution will be evaluated using low-frequency data. In this first step we completely ignore the electrical permittivity. Once this process is done, then the inversion of electrical permittivity can be done in two ways. The frequency band is now shifted to high frequencies past the transition frequency. In the first approach, we fix the electrical conductivity for the entire model and carry out the inversion only for the permittivity. In this case the inversion process is very similar to the one used for the conductivity inversion. The second approach is somewhat more involved, in which the conductivity distribution is used as a constraint rather than as fixed values. So, there is an additional term added to the original functional to be minimized.

\section{Personnel Supported}

1) Alex Becker

Professor

University of California, Berkeley

2) Hung-Wen Tseng

Graduate Student

University of California, Berkeley

Now a Post Doc

Lawrence Berkeley National Lab

3) $\mathrm{Ki} \mathrm{Ha} \mathrm{Lee}$

Staff Scientist

Lawrence Berkeley National Lab

4) Yoonho Song

Visiting Scientist

Korea Institute of Geology, Mining and Materials

Korea

5) Hee Joon Kim

Visiting Professor

Pukyong National University

Korea 


\section{Publications}

Frangos, W. and Becker, A., 1998, Magnetic fields of AM band radio broadcast signals at the Richmond Field Station, Lawrence Berkeley National Lab Report LBNL-42654.

Song, Y., Kim, H.J., and Lee, K.H., 1999, High-Frequency EM Impedance Method for Subsurface Imaging, submitted to Geophysics for publication.

- see attached MS file 'Impedance.doc' -

Song, Y., Morrison, H.F., and Lee, K.H., 1987, High frequency electromagnetic impedance for subsurface imaging, Symposium for Application of Geophysics to Engineering and Environmental Problems (SAGEEP), Reno, Nevada.

\section{Interactions}

Lee, K.H., Becker, A., Pellerin, L., and Frangos, W., High frequency impedance measurements for characterization, monitoring and verification efforts, $1^{\text {st }}$ EMSP National Workshop, July 1998, Chicago.

Lee, K.H., Becker, A., and Frangos, W., High frequency impedance measurements for characterization, monitoring and verification efforts, 2nd EMSP National Workshop, April 2000, Atlanta.

Collaboration with U.S. EPA, 1996 - present, Interagency Agreement DW89937618-01-5 on Electrical and EM Methods for Site Characterization.

\section{Transitions}

None

\section{Patents}

'High-Frequency Electric Field Measurement Using a Toroidal Antenna' Provisional Application Ser. No. 60/070,850 filed 01/08/98

\section{Future Work}

To be conducted through the renewed project with Project Number 73776. A brief summary of the scope of work is given in section Project Productivity. 


\section{Literature Cited}

Alumbaugh, D.L. and Newman, G.A., 1995, High-frequency 3-D EM modeling using massively parallel computers; presented at PIERS '95, Seattle WA.

Knoll, M.D., 1996, A petrophysical basis for ground penetrating radar and very early time electromagnetics: electrical properties of sand-clay mixtures: Ph.D. dissertation, Univ. Brit. Columbia, July

Lee, K. H., Xie, G., Hoversten, G. M., and Pellerin, L., 1995, EM imaging for environmental site characterization, International Symposium on Three-dimensional Electromagnetics. Oct. 4-6, Schlumberger-Doll Research, Ridgefield, CT, USA.

Lee, K.H., 1997, High-frequency electric field measurement using a toroidal antenna, Ernest Orlando Lawrence Berkeley National Laboratory, LBNL-39894, UC-2040.

Mackie, R.L., and Madden, T.R., 1993, Three-dimensional magnetotelluric inversion using conjugate gradients, Geophys. J. Int., 115, 215-229.

Newmann, G.A., and Alumbaugh, D., 1997, Three-dimensional massively parallel electromagnetic inversion - I. Theory:, Geophys. J. Int., 128, 345-354.

Newmann, G.A., 3D Magnetotelluric modeling and inversion, The Second International Symposium on Three-Dimensional Electromagnetics (3DEM-2), Univ. of Utah, Oct.26-29, 1999.

Pellerin L., Labson, V.F., Pfeifer, M.C., 1995, VETEM - A Very early Time-domain electromagnetic system - the first year; In the proceedings of the Symposium on the Application of Geophysics to Engineering and Environmental Problems (SAGEEP).

Pellerin L., Alumbaugh, D.L., Pfeifer, M.C., 1997, The Electromagnetic Integrated Demonstration at the INEL Cold Test Pit; In the proceedings of the Symposium on the Application of Geophysics to Engineering and Environmental Problems (SAGEEP), Reno, Nevada, March 23-26

Persoff, P., Moridis, G.J., James, A., and Frangos, W., 1996, Laboratory study of closure cap repair techniques: LBNL report LBNL-39420, Dec.

Sasaki, Y., 3-D inversion of electrical and electromagnetic data on PCs, The Second International Symposium on Three-Dimensional Electromagnetics (3DEM-2), Univ. of Utah, Oct.26-29, 1999.

Sternberg, B.K., and Poulton, M.M., 1996, High-resolution subsurface imaging and neural network recognition: non-intrusive buried substance location, topical report: DOE Contract No. DE-AC2192MC29101 A0001 
Stewart, D. C., Anderson, W. L., Grover, T. P. and Labson, V. F., 1994, Shallow subsurface mapping by electromagnetic sounding in the $300 \mathrm{kHz}$ to $30 \mathrm{MHz}$ range: model studies and prototype system assessment: Geophysics, 59, pp. 1201-1210

Vozoff, K., ed., 1986, Magnetotelluric Methods: Geophysics Reprint Series No. 5, Society of Exploration Geophysicists

Wait, J.R., 1995, Excitation of a conducting half-space by a toroidal coil: IEEE Antennas and Propagation Magazine, v.37, pp.72-74.

Wright, D.L., Grover, T.P., Labson, V.F., and Pellerin, L., 1996, The Very Early Time Electromagnetic (VETEM) system: First Field Test Results: Proc. of the Symposium on the application of geophysics to engineering and environmental problems (SAGEEP), April 28 - May 1, 1996, Keystone, Colorado. Zhang, Z., and Oldenburg, D.W., 1999, Simultaneous reconstruction of 1-D susceptibility and conductivity from electromagnetic data, Geophysics, 64, 33-47. 